\title{
PRÉ-CONCENTRAÇÃO DE CARVÃO MINERAL ASSISTIDO POR SENSOR DE RAIOS-X EM EQUIPAMENTO SORTING*
}

\author{
Moacir Medeiro Veras ${ }^{1}$ \\ Aaron Samuel Young ${ }^{2}$ \\ Anderson Luis Weiss ${ }^{3}$ \\ Gerson Luis Miltzarek ${ }^{4}$ \\ Marina Signor Castaman ${ }^{5}$ \\ Fernando Leinhard de Freitas ${ }^{6}$ \\ Carlos Otávio Petter ${ }^{7}$ \\ Carlos Hoffmann Sampaio ${ }^{8}$
}

\section{Resumo}

A tecnologia de sensor-based sorting faz uso de sensores high-tech para préconcentrar seletivamente partículas de minérios que tenham teores toleráveis para planta de beneficiamento, rejeitando as partículas que são totalmente estéreis ou rejeitos. A pré-concentração de carvão assistido pelo método Dual Energy X-Ray Transmition possibilita separar seletivamente o carvão com melhor quantidade de matéria orgânica das rochas silicáticas com maior densidade. $O$ objetivo desse trabalho foi investigar a afinidade da tecnologia de sorting na pré-concentração do carvão mineral da Carbonífera do Cambuí/PR assistido por sensor de raios-x. Os testes foram realizados em dois lotes cujo range granulométrico foi -4"+2" e -2"+1". A pré-concentração foi feita em 2 cortes de separação, o primeiro para retirar o rejeito e o segundo para obter 2 tipos de produtos. Os resultados indicaram que $52,15 \%$ da massa foi rejeitada, concentrando $47,85 \%$ do material de carvão na fração média 2"+1". Na fração grossa -4"+2" foi rejeitado $62,51 \%$ da massa e concentrado $37,49 \%$ de carvão. As análises imediatas dos pré-concentrados mostraram que na fração grossa houve redução de $44,57 \%$ de cinzas totais e houve aumento de $4,59 \%$ para $8,29 \%$ de enxofre [S], enquanto na fração média a redução foi de $36,98 \%$ de cinzas totais e de $1,19 \%$ [S].

Palavras-chave: Pré-concentração; Carvão; Sorting; Sensor; Raios-x.

\section{MINERAL COAL PRECONCETRATION USING X-RAY SENSOR SORTING EQUIPMENT}

\section{Abstract}

Sensor-based sorting is a technology that uses high-tech sensors to selectively separate ore particles which are acceptable to beneficiation plants from material that is totality barren or waste. The pre-concentration of coal assisted by the Dual Energy $X$-Ray Transmission method allows for the selective separation of coal with the best amount of organic matter from silica waste rocks with higher density. The objective of this study is to investigate the affinity of sorting technology for mineral coal preconcentration in Paraná supported by $\mathrm{x}$-ray sensor. Tests were performed in two batches of size ranges $-4 "+2$ " (coarse) and $-2 "+1 "$ (medium). The pre-concentration occurred in two phases of separation, the first was to remove waste and the second was to obtain two product types. The results indicated that $52.15 \%$ of the mass was rejected, concentrating $47.85 \%$ of the coal matter for the medium size range $(-2 "+1$ "). For the coarse size range $(-4 "+2 "), 62.51 \%$ of mass was rejected and $37.49 \%$ of the coal was concentrated. Immediate analyses of the pre-concentrates showed that in the coarse fraction there was a reduction of $44.57 \%$ of ash content and an increase 
from $4.59 \%$ to $8.29 \%$ in Sulphur content, while the medium fraction there was a reduction of $36.98 \%$ in ash and $1.19 \%$ in Sulphur.

Keywords: Preconcentration; Coal; Sorting; Sensor; X-Ray.

1 Tecnologia em materiais, doutorando, professor, LAPROM, IFAP / UFRGS, Porto Alegre, Rio Grande do Sul, Brasil.

2 Engenheiro de minas, mestrando, LAPROM, UFRGS, Porto Alegre, Rio Grande do Sul, Brasil.

3 Engenheiro de minas, Carbonífera do Cambuí, Figueira, Paraná, Brasil.

4 Geólogo, Doutor, LAPROM, UFRGS, Porto Alegre, Rio Grande do Sul, Brasil.

5 Engenheiro de minas, Graduanda, LAPROM, UFRGS, Porto Alegre, Rio Grande do Sul, Brasil.

6 Engenheiro de minas, Mestrando, LAPROM, UFRGS, Porto Alegre, Rio Grande do Sul, Brasil.

7 Engenheiro de Minas, PhD eng., Professor, LAPROM, UFRGS, Porto Alegre, Rio Grande do Sul, Brasil.

8 Engenheiro de Minas, PhD eng., Professor, LAPROM, UFRGS, Porto Alegre, Rio Grande do Sul, Brasil.. 


\section{INTRODUÇÃO}

A matéria carbonosa que constitui o carvão é formada por carbono, oxigênio, nitrogênio, enxofre e traços de outros elementos, consoante afirmações de Carneiro [1]. Essa matéria normalmente está associada, em maior ou menor quantidade, a rochas, como arenito, siltito, folhelhos e diamictitos, e a minerais como a pirita. De certa forma, essa matéria associada é considerada como "impureza" do carvão. Assim sendo, a separação desse material indesejável melhora a qualidade do carvão bruto extraído da mina, também denominado Run-of-Mine (ROM), resultando em uma melhoria no rendimento do carvão no seu uso final como citado por MME [2].

Segundo a nota técnica $\mathrm{n}^{\circ} 034$ da ANEEL [3], o carvão da Carbonífera do Cambuí/PR apresenta intercalação de siltito carbonoso e arenito com 18 a 30\% de cinzas e 7 a $12 \%$ de enxofre em sua composição. No Brasil consoante a leitura de Soares, Santos e Possa [11], os estágios que abrangem o beneficiamento do carvão são: britagem, peneiramento, jigagem, circuito de hidrociclones, espirais concentradoras e em alguns casos flotação.

Rúbio et al. [5] demonstra que concentração de carvão mineral por técnicas conservadoras está se estagnando devido a redução de seus recursos minerais, nos quais o beneficiamento e a extração estão cada vez mais complexos.

A inserção de novas tecnologias de sensores na mineração é uma técnica inovadora no beneficiamento no estágio de pré-concentração. Segundo Young et al. [7] e Peticilla et al. [12], deduzem que no futuro os estágios de beneficiamento precisarão focar no pré-concentrado para garantir melhores teores de minério na alimentação da usina. Sob essa ótica, Veras et al. [6] explica que o setor da mineração pode gerar uma maximização no beneficiamento e uma minimização nos custos com insumos energéticos e ambientais.

Ainda em Young [7], o sensor based sorting (SBS) faz uso de sensores de raios-x ou detector de imagem para identificar espécies de minerais e separá-los seletivamente, permitindo que os equipamentos nos estágios seguintes operem com sua capacidade reduzida.

Segundo Wotruba et al. [8], a tecnologia SBS vem sendo usada com grande eficácia para melhorar o beneficiamento gravimétrico, uma vez que auxiliado como uma etapa de pré-concentração de partículas portadoras de teores toleráveis é possível remover quase toda massa de estéril que iria para a usina.

O Sensor de Dual Energy-X-Ray Transmition (DE-XRT) faz aquisição do nível de atenuação da radiação que atravessa a partícula. Jönsson [9] e em Peticila et al. [12] explicam que os dados do raios-x são enviados para o software que se apropria do sinal oriundo das propriedades de densidade equivalente da partícula e converte-o em uma imagem de cor falsa para simplificar a leitura em cada pixel. Neste sentido, Berwanger e Gaastra [10] complementam colocando que a aquisição do sinal da partícula com equivalência a alta densidade é representada pela cor amarela, e a baixa densidade é representada pela cor azul.

De maneira conexa ao estudo em tela, o trabalho tem o objetivo de explorar as diferenças nas propriedades de densidade do carvão mineral da Carbonífera do Cambuí para avaliar a afinidade da tecnologia de sensor-based sorting na préconcentração assistida por sensor de raios-x. Para tanto, o estudo fará uso de duas faixas granulométricas, onde os ensaios de análise imediata e determinação de enxofre serão realizados em cada estágio da pré-concentração e seus rejeitos. 


\section{MATERIAIS E MÉTODOS}

\subsection{Amostra}

O material usado para este estudo é oriundo de uma mina de carvão pertencente à Carbonífera do Cambuí no Estado do Paraná/BR. Foram recebidas duas amostras de material distinguidas pela granulometria $-2 "+1 "$, denominada de fração média, e outra -4"+2" chamada de fração grossa. A amostragem foi feita pela empresa in situ respeitando o desenvolvimento da lavra subterrânea. O carvão está disposto em uma estrutura plano horizontal em camadas entre $0,60 \mathrm{~m}$ e $1 \mathrm{~m}$ de espessura como ilustra a Figura 1. A camada de carvão está inserida em um siltito maciço cuja altura da camada varia entre 0,6 a $1 \mathrm{~m}$, sendo aplicado um corte na galeria de 1,3m ocasionando uma quantidade de material indesejado para usina resultante do realce de teto necessário para operação. O material ensaiado é, portanto, uma mistura de carvão da camada Cambuí e do siltito proveniente da rocha encaixante sobreposta à camada de Carvão.

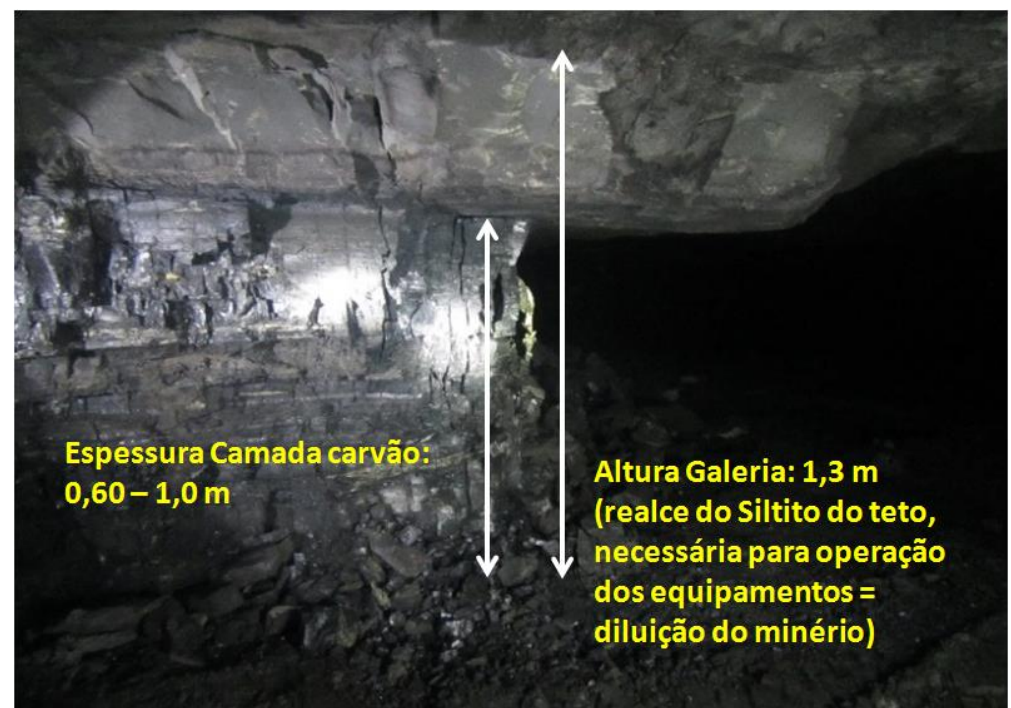

Figura 1. Altura da camada de carvão na mina subterrânea.

As amostras recebidas foram homogeneizadas e quarteadas visando a representatividade e separação de alíquotas como ilustrado na Figura 2. Foram preparadas duas alíquotas, uma de 22 kg na fração -2"+1" e 32,48 kg na fração 4"+2" para testes no equipamento sorting e posterior análise imediatas.

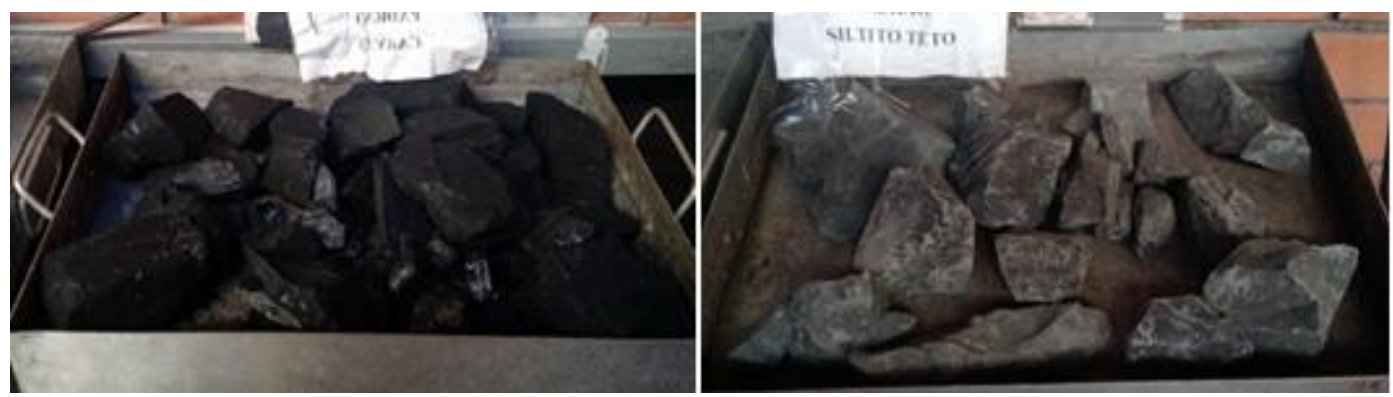

Figura 2. Alíquotas de carvão usadas para testes no equipamento sorting.

\subsection{Sistema de sorting}


Utilizou-se o COMEX Lab-Sorter MSX-400-VL-XR-3D na Universidade Federal do Rio Grande do Sul. Um layout do equipamento de sorting detalhado na Figura 3 é constituído de correia transportadora, porta de alimentação, unidade de câmera óptica, unidade de raios-x, flap de rejeição de material, compressor e central de TI, caixas de recepção de material. Para o ensaio, o material de alimentação é fornecido manualmente onde as partículas individuais são distribuídas na correia transportadora. As rochas são fornecidas para unidade de análise de raios- $\mathrm{x}$ e na extremidade da correia entram na zona do analisador óptico. Nesta área, diferentes propriedades / densidades elementares das partículas são analisadas e registradas usando a tecnologia de sensor X-RAY de alta resolução.

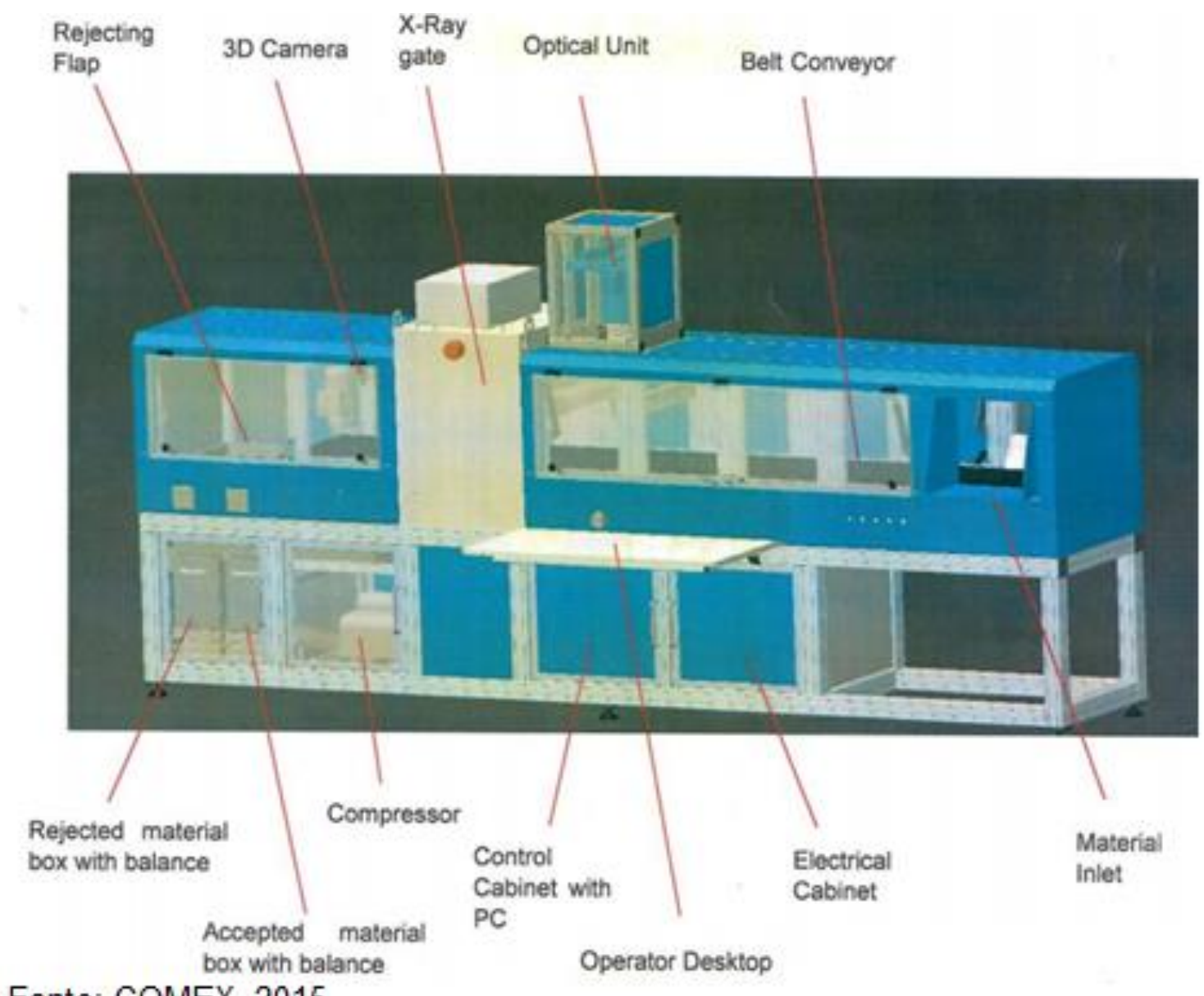

Fonte: COMEX, 2015.

Figura 3. Equipamento COMEX Lab-Sorter MSX-400-VL-XR-3D.

O princípio de funcionamento da tecnologia ilustrado na Figura 4 registra as imagens pela unidade de raios- $x$ em condições dinâmicas. O sistema é operado por uma unidade de controle baseado em um PC industrial que emprega um algoritmo de separação baseado nos critérios de separação selecionados. Cada partícula pode ser identificada em termos da sua densidade equivalente e a sua posição na correia é registrada para possível rejeição adicional. 


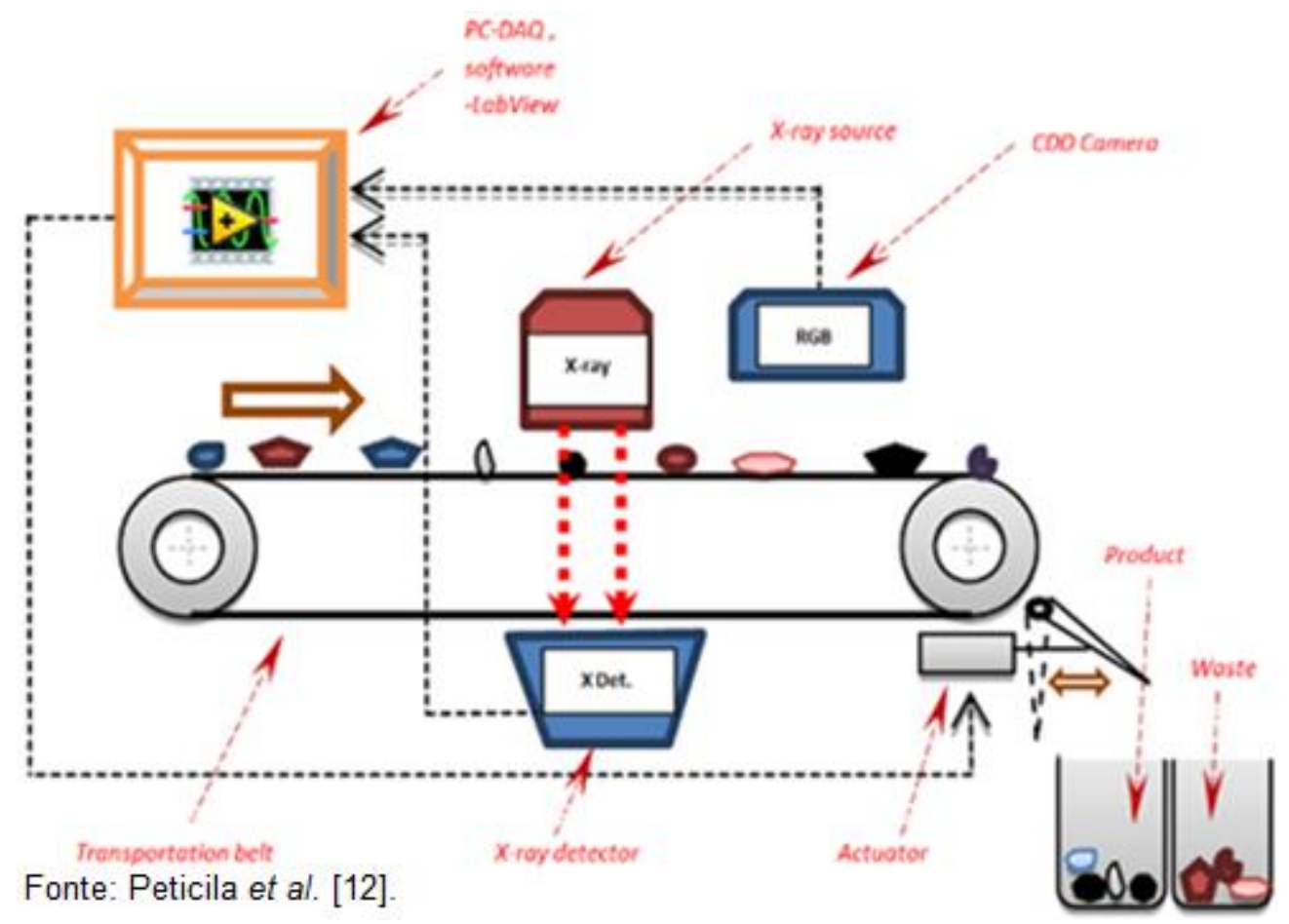

Figura 4. Princípio do sistema de separação XRAY-T CXR.

Os resultados do processamento de imagem podem ser exibidos como um número de diferentes parâmetros para descrever as propriedades físicas do material. Além da densidade do material, existem mais de 50 parâmetros passivos de estudo que compreendem: o diâmetro, perímetro, centro de massa, momento de inércia, orientação das partículas, fator de alongamento, entre outros, normalmente utilizados para a identificação da forma da partícula. Podem também ser utilizadas combinações adicionais destes parâmetros para distinguir as partículas de interesse. Cada partícula tem uma posição e um tamanho especificado, pelo que é possível utilizar o mecanismo de rejeição para separá-los da corrente de material.

\subsubsection{Captura de imagem por sensor DE-XRT}

As partículas de carvão são submetidas ao scan no sensor de dupla energia de transmissão de raios-X (DE-XRT). O sinal de energia registrado pelos sensores carrega informações que identificam a relação de densidade equivalente de cada partícula, onde são enviados ao software para serem tratados e convertidos em pixels para serem representados em uma escala de cor. A medida dessa escala de cor representará uma densidade equivalente ao material de menor densidade quando este apresentar uma cor azul e a cor amarela representa o material de maior densidade equivalente e a preto indica uma densidade acima do permitido pelo equipamento.

A Figura 5 ilustra uma imagem do scan assistido por sensor de raios-x no carvão investigado, onde os dados podem ser interpretados pelo software na forma de cor possibilitando separar seletivamente o produto do rejeito. As partículas no retângulo vermelho são selecionadas pelo software para rejeição. 


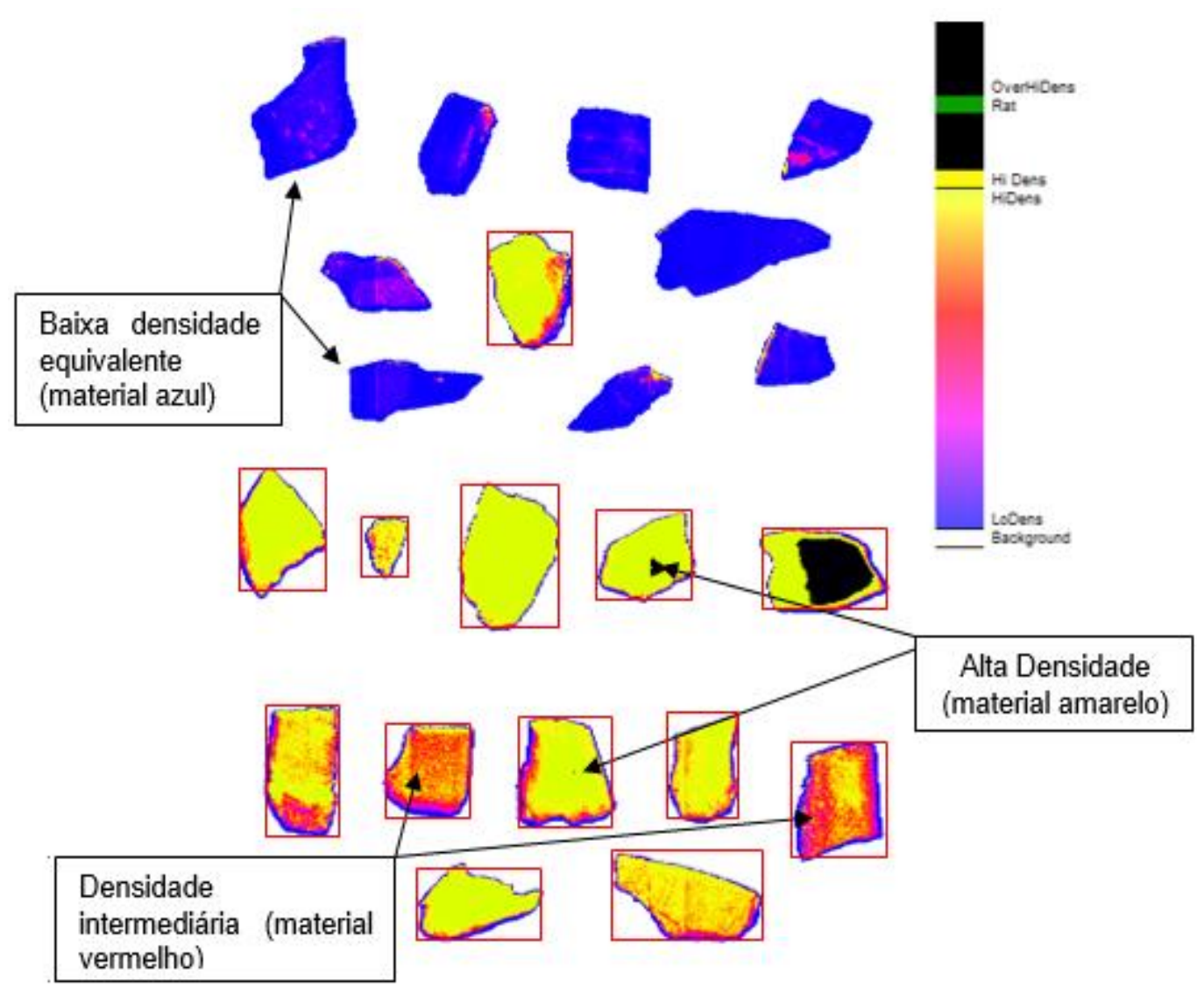

Figura 5. Imagem obtida por sensor de raios-x do material da alimentação.

O material de alimentação fornecido ao nosso sistema de sorting é passado uma partícula de cada vez e separado em produto ou rejeito. Uma vez analisado pelo sensor, o produto e as frações de rejeitos são direcionados para recipientes de amostra. Em seguida, ambas as frações são pesadas e identificadas para análise imediata.

\subsection{Análises imediatas}

A análise imediata compreende as determinações de umidade higroscópica, cinzas, materiais voláteis e carbono fixo. A umidade higroscópica do material foi determinada obedecendo as determinações da ASTM D3173:11, onde aproximadamente $1 \mathrm{~g}$ de carvão pulverizado abaixo de $0,25 \mathrm{~mm}$ (-60mesh) foi colocado em cadinho de porcelana e posto em estufa elétrica durante 60 minutos a uma temperatura de $105^{\circ} \mathrm{C}$.

A quantidade de matérias voláteis foi quantificada por metodologia determinada pela norma ASTM D3175/2011, onde aproximadamente $1 \mathrm{~g}$ de carvão pulverizado abaixo de $0,25 \mathrm{~mm}$ (-60mesh) foi colocado em cadinho de quartzo com tampa e levado ao forno mufla durante 5 minutos a uma temperatura de $900^{\circ} \mathrm{C}$. $\mathrm{O}$ cálculo do teor de matérias voláteis foi feito em duas etapas. Na primeira etapa se calcula a perda de massa.

O método para determinar cinzas totais obedeceu ao disposto na norma ASTM D3174:12, cuja Figura 6 ilustra equipamentos usados para esse ensaio com 0 carvão onde em (a) a balança analítica registra as massas antes e depois da queima e em (b) o forno mufla usado para queima de massas que pode chegar a 
temperaturas de até $1200^{\circ} \mathrm{C}$, onde a amostra é colocada em forno mufla durante 60 minutos a uma temperatura de $800^{\circ} \mathrm{C}$. Carbono fixo foi determinado por diferença da soma de cinzas + voláteis.

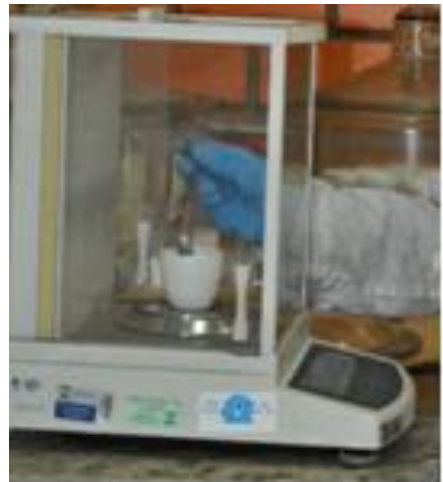

(a)

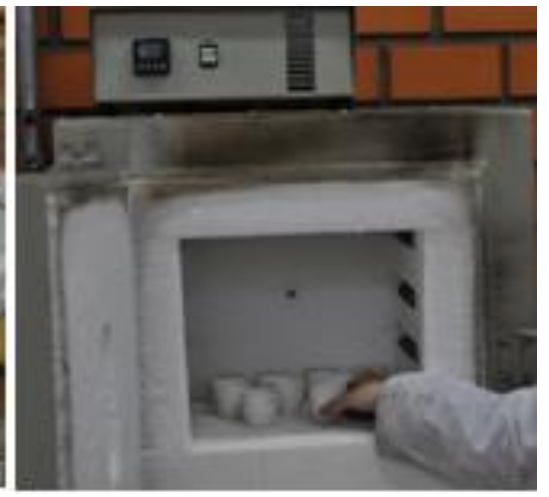

(b)

Figura 6. Equipamentos usados para determinar cinzas totais nas amostras de carvão.

Também foram determinados os teores de enxofre total [S] e a separação densimétrica via afunda/flutua. Os ensaios de afunda-flutua foram feitos nas densidades de corte entre $1,6 \mathrm{~g} / \mathrm{cm}^{3}$ e $2,4 \mathrm{~g} / \mathrm{cm}^{3}$. Os reagentes usados para preparo dos meios densos compreendem o xileno $\left(0.89 \mathrm{~g} / \mathrm{cm}^{3}\right)$, percloroetileno $\left(1.61 \mathrm{~g} / \mathrm{cm}^{3}\right)$ e bromofórmio $\left(2.89 \mathrm{~g} / \mathrm{cm}^{3}\right)$. A Figura 7 apresenta o procedimento do ensaio de afunda-flutua que feito em beaker (1), onde posteriormente o material flutuado é separação em funil/filtrado (2).

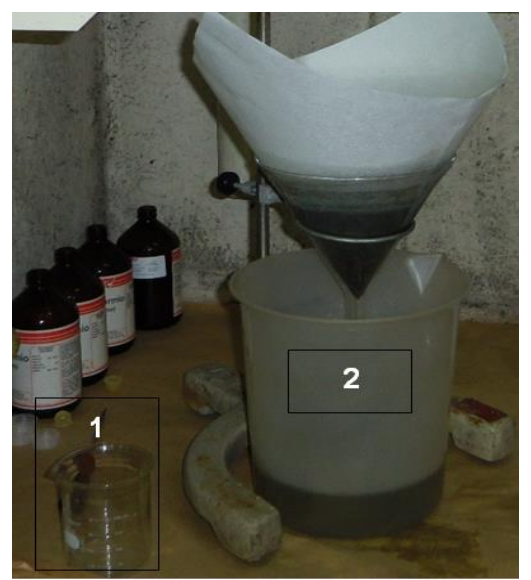

Figura 7. Sistema de separação densitária por técnica de afunda-flutua.

As combinações dos reagentes orgânicos para constituir as faixas de densidade são calculadas pela Equação (1), onde o volume do líquido a acrescentar $\left(\mathrm{V}_{2}\right)$ é resultante do volume de líquido a alterar $\left(\mathrm{V}_{1}\right)$, sendo a expressão a seguir multiplicada pela densidade do líquido a alterar $\left(\rho_{1}\right)$, subtraída pela nova densidade $(\rho)$, e toda essa expressa é dividida pela subtração entre a nova densidade pela densidade do líquido a acrescentar $\left(\rho_{2}\right)$.

$$
\mathbf{V}_{2}=\frac{\mathbf{V}_{1} \cdot\left(\boldsymbol{\rho}_{1}-\rho\right)}{\left(\rho-\rho_{2}\right)}
$$




\section{RESULTADOS E DISCUSSÃO}

Os testes foram realizados nas amostras de carvão separadamente para cada fração investigada, assim os resultados serão apresentados separadamente respeitando as particularidades de operação para o estudo em tela.

\subsection{Carvão médio}

A amostra de carvão médio, fração entre $-2 "+1 "$ foi pré-concentrado consoante a metodologia no fluxograma da Figura 8, cujo balanço de massa representa cada ensaio e análises imediatas que evidenciam as massas da alimentação, concentrados, rejeito, cinzas totais, umidade, matérias voláteis, enxofre [S] e as massas flutuadas na densidade de corte em $1,6 \mathrm{~g} / \mathrm{cm}^{3}$. O primeiro corte (sorter 1 ) objetivou remover o rejeito do carvão, seguido de um segundo corte de enriquecimento (sorter 2), cuja finalidade foi obter um pré-concentrado de melhor qualidade.

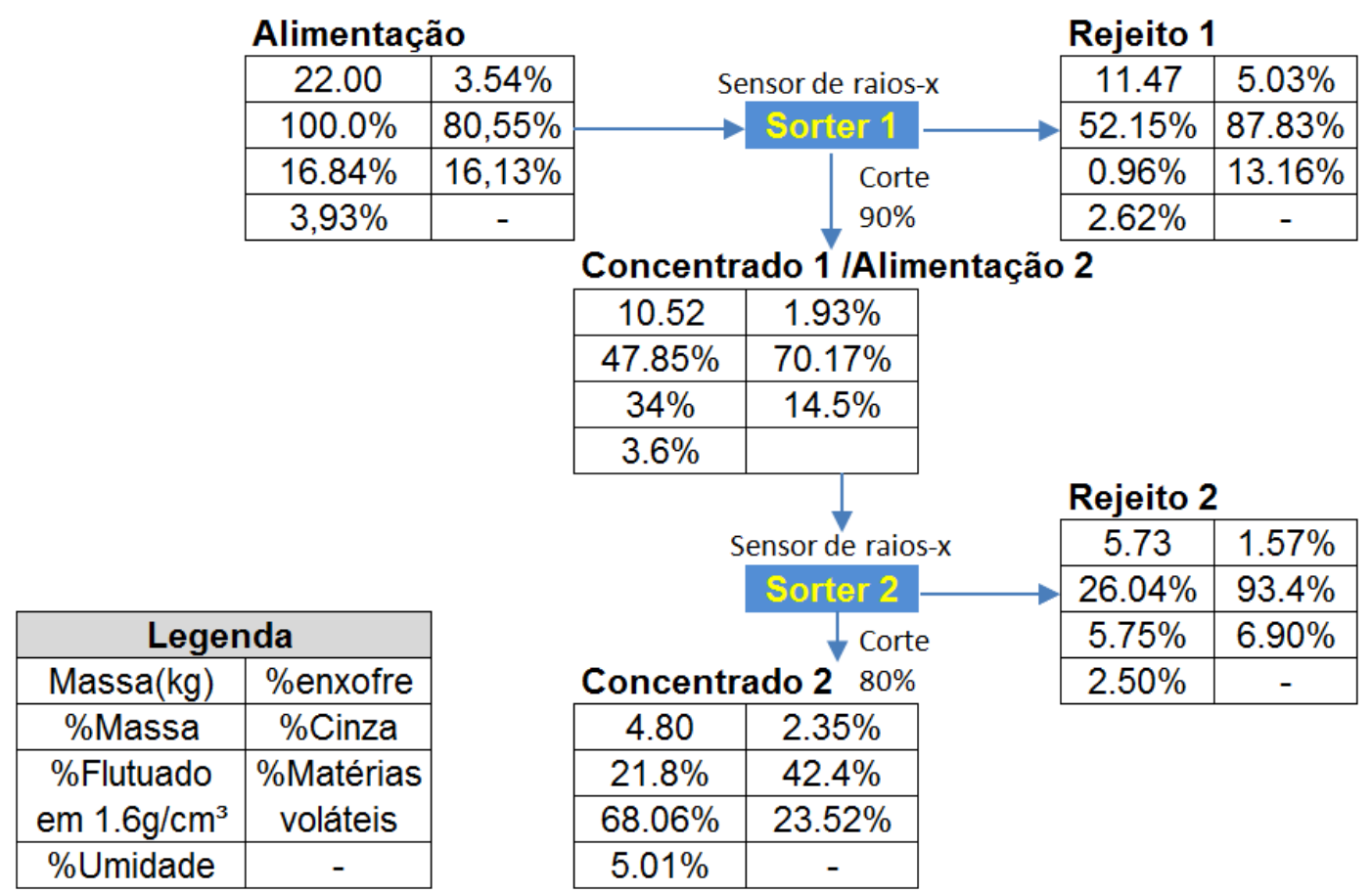

Figura 8. Fluxograma dos testes de pré-concentração com carvão médio.

Os testes de análise no carvão médio mostraram que a pré-concentração via seca por meio da tecnologia de sorting rejeitou aproximadamente $52,15 \%$, concentrando aproximadamente $47,85 \%$ de massa, sendo $34,15 \%$ do minério de carvão com densidade menor a $1,6 \mathrm{~g} / \mathrm{cm}^{3}$ no primeiro corte. No segundo corte, objetivando obter um carvão de melhor qualidade, o concentrado obteve massa de $21,8 \%$ com $68,06 \%$ do carvão com densidade inferior a $1,6 \mathrm{gcm}^{3}$, comparado com a alimentação.

As massas pré-concentradas em sorting ao serem submetidas ao ensaio de afunda flutua apresentaram uma distribuição densitária na Tabela 1 confirmando que quase $80 \%$ da alimentação tem densidade maior que $1,6 \mathrm{~g} / \mathrm{cm}^{3}$, enquanto que os rejeitos 1 e 2 apresentam aproximadamente $96 \%$ e $94 \%$ de massa com densidade maior que 
$1,6 \mathrm{~g} / \mathrm{cm}^{3}$. Por outro lado, as massas concentradas 1 e 2 apresentaram $34 \%$ e $68 \%$ de suas massas menor que $1,6 \mathrm{~g} / \mathrm{cm}^{3}$ respectivamente.

Tabela 1. Range das massas e percentuais do ensaio de afunda flutua do carvão médio.

\begin{tabular}{|c|c|c|c|c|c|c|c|c|c|c|}
\hline \multirow{2}{*}{$\begin{array}{c}\text { Meio denso } \\
\left(\mathrm{g} / \mathrm{cm}^{3}\right)\end{array}$} & \multicolumn{2}{|c|}{ Alim* } & \multicolumn{2}{|c|}{ Rejeito 1} & \multicolumn{2}{|c|}{ Conc 1/Alim 2* } & \multicolumn{2}{|c|}{ Conc 2* } & \multicolumn{2}{|c|}{ Rejeito 2} \\
\hline & $(\mathrm{kg})$ & (\%) & (kg) & (\%) & (kg) & (\%) & (kg) & (\%) & (kg) & (\%) \\
\hline$f 1,6$ & 3.44 & $16.7 \%$ & 0.10 & $1.0 \%$ & 3.34 & $34 \%$ & 3.03 & $68 \%$ & 0.30 & $6 \%$ \\
\hline$f 1,7$ & 0.19 & $0.9 \%$ & 0.06 & $0.6 \%$ & 0.13 & $1 \%$ & 0.09 & $2 \%$ & 0.04 & $1 \%$ \\
\hline$f 1,8$ & 0.29 & $1.4 \%$ & 0.00 & $0.0 \%$ & 0.29 & $3 \%$ & 0.21 & $5 \%$ & 0.08 & $2 \%$ \\
\hline$f 1,9$ & 0.29 & $1.4 \%$ & 0.08 & $0.7 \%$ & 0.22 & $2 \%$ & 0.08 & $2 \%$ & 0.14 & $3 \%$ \\
\hline$f 2,0$ & 0.24 & $1.2 \%$ & 0.01 & $0.1 \%$ & 0.24 & $2 \%$ & 0.10 & $2 \%$ & 0.14 & $3 \%$ \\
\hline$f 2,2$ & 0.40 & $1.9^{\circ}$ & 0.0 & $0.8 \%$ & 0.3 & $3 \%$ & 0.1 & $3 \%$ & 0.19 & $4 \%$ \\
\hline$f 2,4$ & 0.70 & $3.4 \%$ & 0.22 & $2.0 \%$ & 0.48 & $5 \%$ & 0.24 & $5 \%$ & 0.24 & $5 \%$ \\
\hline A 2,4 & $15.0^{-}$ & $73.0 \%$ & 10.30 & $94.9 \%$ & 4.71 & $48 \%$ & 0.58 & $13 \%$ & 4.13 & $79 \%$ \\
\hline$\Sigma$ & 20.5 & $100 \%$ & 10.85 & $100 \%$ & 9.71 & $100 \%$ & 4.46 & $100 \%$ & 5.25 & $100 \%$ \\
\hline
\end{tabular}

*Alim - Alimentação; Conc 1/Alim 2 - Concentrado 1/Alimentação 2; Conc 2 - Concentrado 2.

Quanto aos dados de análises, imediatas, físicas e elementares, no rejeito 1, podese notar que houve aumento de aproximadamente $8 \%$ de cinzas totais, redução de $3 \%$ de matérias voláteis, $16 \%$ massa flutuada em $1,6 \mathrm{~g} / \mathrm{cm}^{3}$ e $1,31 \%$ de umidade cujos os valores estão apresentados na Tabela 2. Também o teor de enxofre teve aumento de $1,5 \%$.

Tabela 2. Dados de análise imediata, enxofre, e afunda-flutua do carvão médio.

\begin{tabular}{|c|c|c|c|c|c|c|}
\hline \multirow{2}{*}{$\begin{array}{l}\text { Pré- } \\
\text { concentrado }\end{array}$} & \multicolumn{4}{|c|}{ Análise imediata ${ }^{\text {iu }}$} & \multirow{2}{*}{$\begin{array}{c}\begin{array}{c}\text { Análise } \\
\text { elementar }\end{array} \\
\text { Enxofre (S) }\end{array}$} & \multirow{2}{*}{$\begin{array}{c}\text { Análise } \\
\text { densitária } \\
\text { Massa flutuada } \\
\left(1,6 \mathrm{~g} / \mathrm{cm}^{3}\right)\end{array}$} \\
\hline & Umidade & Cinzas & $\begin{array}{c}\text { Matéria } \\
\text { Volátil }\end{array}$ & $\begin{array}{c}\text { Carbono } \\
\text { fixo* }\end{array}$ & & \\
\hline Alimentação & $3,93 \%$ & $81 \%$ & $13,79 \%$ & $1,73 \%$ & $3,54 \%$ & $16,84 \%$ \\
\hline Rejeito 1 & $2,62 \%$ & $88 \%$ & $13,16 \%$ & $0,00 \%$ & $5,03 \%$ & $0,96 \%$ \\
\hline Conc 1/Alim 2 & $5,36 \%$ & $73 \%$ & $14,47 \%$ & $7,56 \%$ & $1,93 \%$ & $34,15 \%$ \\
\hline Rejeito 2 & $2,50 \%$ & $93 \%$ & $6,90 \%$ & $0,00 \%$ & $1,57 \%$ & $5,75 \%$ \\
\hline Conc2 & $5,01 \%$ & $42 \%$ & $23,52 \%$ & $29,07 \%$ & $2,35 \%$ & $68,06 \%$ \\
\hline
\end{tabular}

*obtido pela equação (100-Cinzas-Matéria Volátil).

iu (Isento de umidade)

\subsection{Carvão grosso}

A amostra de carvão grosso (fração entre -4"+2"), também foi pré-concentrado em dois cortes, cujos valores de balanço de massa e dados das análises imediatas estão apresentados no fluxograma da Figura 9, onde a apresentação dos dados obedeceu a mesma metodologia do fluxograma no item 3.1. O primeiro corte (sorter 1) objetivou remover o rejeito do carvão, e segundo corte (sorter 2) teve finalidade de obter um pré-concentrado com melhor qualidade. 


\begin{tabular}{|c|c|c|c|c|}
\hline \multicolumn{2}{|c|}{ Alimentação } & \multirow[b]{2}{*}{ Sens } & \multicolumn{2}{|c|}{ Rejeito 1} \\
\hline 32,48 & \begin{tabular}{|l|}
$4,59 \%$ \\
\end{tabular} & & 20,30 & $2,96 \%$ \\
\hline $100,0 \%$ & \begin{tabular}{|l|}
$71,79 \%$ \\
\end{tabular} & $\rightarrow$ Sorter 1 & $62,51 \%$ & $91,70 \%$ \\
\hline $24,14 \%$ & $14,48 \%$ & Corte & $0,04 \%$ & $8,82 \%$ \\
\hline $3,88 \%$ & - & $90 \%$ & $2,89 \%$ & - \\
\hline
\end{tabular}

Concentrado 1/Alimentação 2

\begin{tabular}{|c|c|}
\hline 12,18 & $7,30 \%$ \\
\hline $37,49 \%$ & $38,60 \%$ \\
\hline $64,33 \%$ & $23,93 \%$ \\
\hline $5,52 \%$ & - \\
\hline
\end{tabular}

\begin{tabular}{|c|c|}
\hline \multicolumn{2}{|c|}{ Legenda } \\
\hline Massa(kg) & $\%$ enxofre \\
\hline$\%$ Massa & $\%$ Cinza \\
\hline$\%$ Flutuado & $\%$ Matérias \\
em $1.6 \mathrm{~g} / \mathrm{cm}^{3}$ & voláteis \\
\hline$\%$ Umidade & - \\
\hline
\end{tabular}

Sensor de raios-x

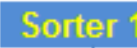

Rejeito 2

Figura 9. Fluxograma dos testes de pré-concentração com carvão grosso.

Os testes de análise no carvão grosso mostraram que a pré-concentração por meio de sorting rejeitou aproximadamente $62 \%$ da massa, concentrando aproximadamente $37,49 \%$ de minério de carvão com $64,33 \%$ da massa de carvão com densidade menor que $1,6 \mathrm{~g} / \mathrm{cm}^{3}$. No segundo corte, pré-concentrou $25,10 \%$ da massa elevando para $86,78 \%$ a massa de carvão com densidade menor que $1,6 \mathrm{~g} / \mathrm{cm}^{3}$.

As massas pré-concentradas em sorting foram submetidas ao ensaio de afunda flutua e apresentaram uma distribuição densitária na Tabela 3, cuja alimentação tem $24 \%$ de massa com densidade menor que $1,6 \mathrm{~g} / \mathrm{cm}^{3}$, enquanto que os rejeitos 1 e 2 apresentam mais de $97 \%$ e $80 \%$ dessa massa com densidade maior que $1,6 \mathrm{~g} / \mathrm{cm}^{3}$. Por outro lado, as massas concentradas 1 e 2 pré-concentrou $67 \%$ e $87 \%$ de suas do carvão com densidade menor que $1,6 \mathrm{~g} / \mathrm{cm}^{3}$.

Tabela 3. Range das massas e percentuais do ensaio de afunda flutua do carvão grosso.

\begin{tabular}{c|cc|cc|ccc|cc|cc}
\hline Meio denso & \multicolumn{2}{c}{ Alim $^{\star}$} & \multicolumn{3}{c}{ Rejeito 1 } & \multicolumn{3}{c}{ Conc 1/Alim 2* } & \multicolumn{2}{c}{ Conc 2* } & \multicolumn{2}{c}{ Rejeito 2 } \\
\hline $\mathbf{( g / c m}^{\mathbf{3}} \mathbf{)}$ & $\mathbf{( g )}$ & $\mathbf{( \% )}$ & $\mathbf{( g )}$ & $\mathbf{( \% )}$ & $\mathbf{( g )}$ & $\mathbf{( \% )}$ & $\mathbf{( g )}$ & $\mathbf{( \% )}$ & $\mathbf{( g )}$ & $\mathbf{( \% )}$ \\
\hline $\mathbf{f ~ 1 , 6}$ & 7,29 & $24 \%$ & 0,01 & $0 \%$ & 7,28 & $67 \%$ & 6,67 & $87 \%$ & 0,61 & $19 \%$ \\
\hline $\mathbf{f ~ 1 , 7}$ & 1,37 & $4 \%$ & 0,00 & $0 \%$ & 1,37 & $13 \%$ & 0,68 & $9 \%$ & 0,69 & $21 \%$ \\
\hline $\mathbf{f ~ 1 , 8}$ & 0,21 & $1 \%$ & 0,00 & $0 \%$ & 0,21 & $2 \%$ & 0,21 & $3 \%$ & 0,00 & $0 \%$ \\
\hline $\mathbf{f ~ 1 , 9}$ & 0,05 & $0 \%$ & 0,00 & $0 \%$ & 0,05 & $0 \%$ & 0,05 & $1 \%$ & 0,00 & $0 \%$ \\
\hline $\mathbf{f ~ 2 , 0}$ & 0,06 & $0 \%$ & 0,00 & $0 \%$ & 0,06 & $1 \%$ & 0,05 & $1 \%$ & 0,01 & $0 \%$ \\
\hline $\mathbf{f ~ 2 , 2}$ & 0,83 & $3 \%$ & 0,01 & $0 \%$ & 0,82 & $8 \%$ & 0,01 & $0 \%$ & 0,81 & $25 \%$ \\
\hline $\mathbf{f ~ 2 , 4}$ & 0,61 & $2 \%$ & 0,60 & $3 \%$ & 0,01 & $0 \%$ & 0,01 & $0 \%$ & 0,00 & $0 \%$ \\
\hline $\mathbf{A ~ 2 , 4}$ & 20,13 & $66 \%$ & 18,99 & $97 \%$ & 1,14 & $10 \%$ & 0,02 & $0 \%$ & 1,12 & $34 \%$ \\
\hline$\Sigma$ & $\mathbf{3 0 , 5 4}$ & $\mathbf{1 0 0 \%}$ & $\mathbf{1 9 , 6 1}$ & $\mathbf{1 0 0} \%$ & $\mathbf{1 0 , 9 3}$ & $\mathbf{1 0 0} \%$ & $\mathbf{7 , 6 8}$ & $\mathbf{1 0 0} \%$ & $\mathbf{3 , 2 5}$ & $\mathbf{1 0 0 \%}$ \\
\hline
\end{tabular}

*Alim - Alimentação; Conc 1/Alim 2 - Concentrado 1/Alimentação 2; Conc 2 - Concentrado 2.

Os ganhos e perdas das massas pré-concentradas e determinadas na análise imediata estão apresentados na Tabela 4. Pode-se notar que a umidade, os teores 
de enxofre e matérias voláteis aumentaram nas massas dos concentrados, observando significativos ganhos de carvão com densidade menor que $1,6 \mathrm{~g} / \mathrm{cm}^{3}$. Os teores de cinzas também aumentaram nos rejeitos 1 e 2 reduzindo significativamente nas massas concentradas.

Tabela 4. Resultado da análise imediata, enxofre e afunda-flutua.

\begin{tabular}{|c|c|c|c|c|c|c|}
\hline \multirow{2}{*}{$\begin{array}{l}\text { pré- } \\
\text { concentração }\end{array}$} & \multicolumn{4}{|c|}{ Análise imediata ${ }^{i u}$} & \multirow{2}{*}{$\begin{array}{c}\begin{array}{c}\text { Análise } \\
\text { elementar }\end{array} \\
\text { Enxofre (S) }\end{array}$} & \multirow{2}{*}{$\begin{array}{c}\text { Análise } \\
\text { densitária } \\
\text { Massa flutuada } \\
\left(1,6 \mathrm{~g} / \mathrm{cm}^{3}\right) \\
\end{array}$} \\
\hline & Umidade & Cinzas & $\begin{array}{c}\text { Matéria } \\
\text { volátil }\end{array}$ & $\begin{array}{c}\text { Carbono } \\
\text { fixo* }\end{array}$ & & \\
\hline Alimentação & $3,88 \%$ & & 14,48 & $13,73 \%$ & & $24,14 \%$ \\
\hline Rejeito 1 & 0 & ,70\% & 8 & $0,00 \%$ & $\%$ & $0,04 \%$ \\
\hline Conc 1/ alim2 & $5,52 \%$ & $38,60 \%$ & $23,93 \%$ & $37,47 \%$ & $7,30 \%$ & $64,33 \%$ \\
\hline Conc2 & $6,16 \%$ & $27,22 \%$ & $28,31 \%$ & $44,47 \%$ & $8,29 \%$ & $86,78 \%$ \\
\hline Rejeito 2 & $4,23 \%$ & $61,25 \%$ & $15,05 \%$ & $23,70 \%$ & $5,31 \%$ & $18,89 \%$ \\
\hline
\end{tabular}

*obtido pela equação (100-Cinzas-Matéria Volátil).

iu (Isento de umidade)

\section{CONCLUSÃo}

Os testes de separação com equipamento sorting foram realizados em 2 duas amostras de carvão. A pré-concentração foi realizada na amostra de carvão médio (fração de tamanho +1" -2") e carvão grosso (fração de tamanho +2"-4"). A finalidade deste trabalho foi avaliar a afinidade da tecnologia para separar seletivamente as amostras de carvão.

Os testes de separação foram realizados no sistema COMEX Lab-Sorter MSX-400VL-XR-3D que usou flap pneumático para separar o material, onde a préconcentração foi feita em 2 cortes de separação, o primeiro para retirar o rejeito e o segundo para obter um carvão de melhor qualidade.

Essa metodologia foi usada nas duas amostras, porém com configurações diferentes. Para amostra na fração $-2 "+1 "$, os resultados de laboratório indicaram que $52,15 \%$ foi rejeitado e concentrou $47,85 \%$ do material de carvão médio da alimentação. Na fração -4"+2" (carvão grosso) foi rejeitado $62,51 \%$ e concentrado $37,49 \%$ do material da alimentação.

Os testes de afunda-flutua foram realizados em todas as amostras considerando o material flutuado em $1,6 \mathrm{~g} / \mathrm{cm}^{3}$ para o carvão de boa qualidade. O carvão médio apresentou $16,84 \%$ de sua massa flutuada no ROM. A primeira separação obteve $34,15 \%$ de massa flutuada. Na segunda separação, denominada de enriquecimento, a massa do concentrado 2 flutuou 68,03 \%.

O carvão grosso apresentou $24,14 \%$ de massa flutuada no ROM. Na primeira separação o concentrado 1 obteve $64,33 \%$ da massa flutuada. A segunda separação, denominada de enriquecimento, o concentrou $86,78 \%$ de massa flutuada.

Pode-se inferir que nas frações -4"+2" o equipamento tem sensibilidade para separar o carvão dos contaminantes originários das encaixantes. Nesta granulometria não há ainda a liberação do enxofre presente na camada de carvão. Na fração -2"+1" alguma liberação está presente, permitindo a separação de outros componentes tais como o enxofre pirítico.

Dessa maneira conclui-se que a tecnologia de sensor-based sorting pode ser uma alternativa futura para potencializar a pré-concentração via seca de carvão. 
Dependendo da granulometria de alimentação pode servir para rejeito prévio de rochas alheias a camada de carvão ou, com alimentação em menor granulometria, descartar também parte do enxofre presente na camada de carvão. Com a inclusão desta etapa pode-se assim maximizar as rotinas das usinas e/ou lavras do minério em tela.

\section{Agradecimentos}

Gostaríamos de expressar nossos agradecimentos à Carbonífera do Cambuí, em especial ao Eng. Anderson Luis Weiss, LAPROM, IFAP, CNPq e aos demais colaboradores, pois nesta ação conjunta foi possível frutificar o desenvolvimento desse trabalho.

\section{REFERÊNCIAS}

1 Carneiro. LFS. Avaliação do comportamento da mistura de casca de arroz e carvão mineral na granulometria de 100 a 200 mesh para sua injeção em altos-fornos. Monografia. UFOP. 2012.

2 MME-Ministério de Minas e Energia. Plano nacional de energia 2030. v 6. Epe. 2007.

3 ANEEL. A Situação da Produção de Carvão Mineral no Estado do Paraná. Nota técnica $n^{\circ}$ 034/2011. SGR/ANEEL. junho. 2011.

4 Soares PSM, Santos MDC e Possa MV. Carvão brasileiro tecnologia e meio ambiente. Rio de Janeiro: CETEM/MCT. 2008.

5 Rubio J. Carvão mineral: caracterização e beneficiamento. v 1. Porto Alegre. Nova Linha Artes Gráficas. 1988.

6 Veras MM, Young AS, Petter $\mathrm{CO}$ e Sampaio $\mathrm{CH}$. A mining breakthrough: preconcentration by sensor-based sorting. Mining engineering. $v$ 68. Issue 3 . March 2016:38-42.

7 Young AS, Veras MM, Petter CO e Sampaio $\mathrm{CH}$. Revolution technology x-ray, ccd cameras and sensors in ore processing stages. World Mining Congress. October 1821:2016.

8 Wotruba H, Knapp H, Neubert K, and Schropp C. "Anwendung der sensorgestützten Sortierung für die Aufbereitung mineralischer Rohstoffe," Chemie Inginieur Techik, 86 (6), 2014:773-783.

9 Jönsson, C. Sensor-Based Sorting Technology: Implementation in the mining and mineral processing industry. 2014. Disponível em:

http://www.pm.Ith.se/fileadmin/pm/Exjobb/Exjobb_2014/Joensson_Christian/Article_Ch ristian_Joensson.pdf.

10 Berwanger M, Gaastra M. "Technical and physical principles of sensor technologies applied in the raw materials industry", 2014:47-199. In: Aachen, RWTH, chapter 4. Nienhaus K, Pretz, T, Wotruba H. Sensor Technologies: impulses for the raw Materials Industry. 2014.

11 Possa MV, Santos MDC, Soares PSM. Carvão Brasileiro: tecnologia e meio ambiente. Rio de Janeiro: CETEM/MCT, 2008. Disponível em: <http://www.cetem.gov.br/publicacao/CTs/CT2008-094-00.pdf>

12 Peticila M, Young AS, Cazacliu B, Sampaio CH, Veras MM, Petter CO. Improving the characteristics of recycled aggregates by using mineral processing equipment: water jig and sensor-based sorting. 5th International Conference on Sustainable Solid Waste Management, Athens, Grecy, 21-24 ${ }^{\text {th }}$ June, 2017. 